\title{
STEADY TEARING MODE INSTABILITIES WITH A RESISTIVITY DEPENDING ON A FLUX FUNCTION
}

\author{
Atanda Boussari ${ }^{1}$, Erich Maschke ${ }^{2}$ And Bernard Saramito ${ }^{1}$
}

\begin{abstract}
We consider plasma tearing mode instabilities when the resistivity depends on a flux function $(\psi)$, for the plane slab model. This problem, represented by the MHD equations, is studied as a bifurcation problem. For so doing, it is written in the form $(I(\cdot)-T(S,))=$.0 , where $T(S,$. is a compact operator in a suitable space and $S$ is the bifurcation parameter. In this work, the resistivity is not assumed to be a given quantity (as usually done in previous papers, see [1,2,5,7-10], but it depends non linearly of the unknowns of the problem; this is the main difficulty, with new mathematical results. We also develop in this paper a $1 \mathrm{D}$ code to compute bifurcation points from the trivial branch (equilibrium state).
\end{abstract}

AMS Subject Classification. 34A47, 76E25, 82D10, 35J45.

Received: May 6, 1998. Revised: February 11, 1999.

\section{INTRODUCTION}

We are interested in this study by steady plasma tearing mode instabilities which are of great interest in research on confined plasmas inside a tokamak.

The major difficulty in plasma confining process is the rich variety of instabilities which can appear.

Some instabilities can completely destroy the confinement and must be avoided; other ones, such as tearing mode instabilities, can't be avoided and are responsible of anomalous transport. Their nonlinear behaviour has then to be studied.

These last years, many authors have been interested by these instabilities.

Saramito and Maschke [10] applied bifurcation theory to the study of nonlinear tearing modes, and obtained a bifurcation to a nonlinearly saturated steady state when equilibrium loses stability. Grauer [5] considers interaction of two near marginal modes in presence of $\mathcal{O}(2)$ symmetry, Chen and Morrison [2] also study interaction of two marginal tearing modes but in presence of equilibrium flow. In both works, Grauer [5], Chen and Morrison [2], employed center manifold reduction. Parker [7] solved numerically the full viscoresistive MHD equations and studied bifurcations between the nonlinear states using the periodicity length as a bifurcation parameter.

In all mentioned works, the resistivity is considered either as a constant or as a given quantity depending only on the space variable $x$. But in general, the resistivity depends on the plasma's temperature $T_{e}$. Due to the high diffusion coefficient of this temperature in the parallel direction to the magnetic field, $T_{e}$ can be considered

Keywords and phrases. Bifurcation, tearing modes, MHD instabilities.

1 Laboratoire de Mathématiques Appliquées, Université Blaise Pascal de Clermont-Ferrand, 63177 Aubière Cedex, France.

e-mail: saramito@ucfma.univ-bpclermont.fr

2 C.E.N. Cadarache, 13108 Saint-Paul-Lez-Durance, France. 
as constant per magnetic surface (which is modelized by the equation $\psi=$ constant). We then consider the resistivity as depending on a flux function. Then, when we consider that the resistivity depends nonlinearly on the unknowns of the problem, new mathematical difficulties arise in proving bifurcation theorems, as it will appear in this paper (Lem. 2, Prop. 1, Th. 1 and Cor. 1).

We'll first give equations describing tearing mode instabilities, next we write the considered problem in a functional equation form in a suitable functional space and we end this work by describing a 1D code to compute bifurcation points from the trivial branch (equilibrium state).

\section{EqUATIONS}

Let $\Omega$ be the open set ] $-1 / 2,1 / 2[\times] 0, L[\ni(x, y)$; equations modelizing the tearing mode instabilities in $\Omega$ can be written after normalization

$$
\left\{\begin{array}{l}
\operatorname{div} V=S_{e} \\
\frac{\partial}{\partial t} V+V \cdot \nabla V-\wp_{R} \Delta V+S^{2}(\nabla P-B \cdot \nabla B)=0 \\
\frac{\partial}{\partial t} B+\operatorname{curl}(\eta(\psi) \operatorname{curl} B)-\operatorname{curl}(V \times B)=0 \\
\operatorname{div} B=0
\end{array}\right.
$$

In these equations, $V$ is the velocity, $B$ the magnetic field, $P$ the plasma pressure, $\eta$ the (normalized) resistivity, $\psi$ a flux function $\left(B=e_{z} \times \nabla \psi\right), \wp_{R}$ the Prandl number and $S$ the Lundquist number; $S_{e}$ is a given quantity (with value 0 in the numerical results presented in paragraph 4 below).

Let us assume that there exists known quantities $V_{\mathrm{eq}}, B_{\mathrm{eq}}, P_{\mathrm{eq}}$, and $\psi_{\mathrm{eq}}$ in $C^{\infty}(\bar{\Omega})\left(B_{\mathrm{eq}}=e_{z} \times \nabla \psi_{\mathrm{eq}}\right)$ which represent equilibrium state and verify equations (1), with periodicity of all derivatives in the y direction (generally, these quantities only depend of $x$ ).

For particular values of the parameters, this equilibrium state becomes unstable. To study bifurcated branches we consider a perturbation of the equilibrium state . Let $\hat{V}, \hat{B}, \hat{\psi}, \hat{P}$ be defined by

$$
V=V_{\text {eq }}+S \hat{V}, \psi=\psi_{\text {eq }}+\hat{\psi}, B=B_{\text {eq }}+\hat{B}, \hat{B}=e_{z} \times \nabla \hat{\psi}, P=P_{\text {eq }}+\frac{1}{S} \hat{P} .
$$

From the equations (1) for $V, B, P$, we obtain the following equations for $\hat{V}, \hat{B}, \hat{P}$,

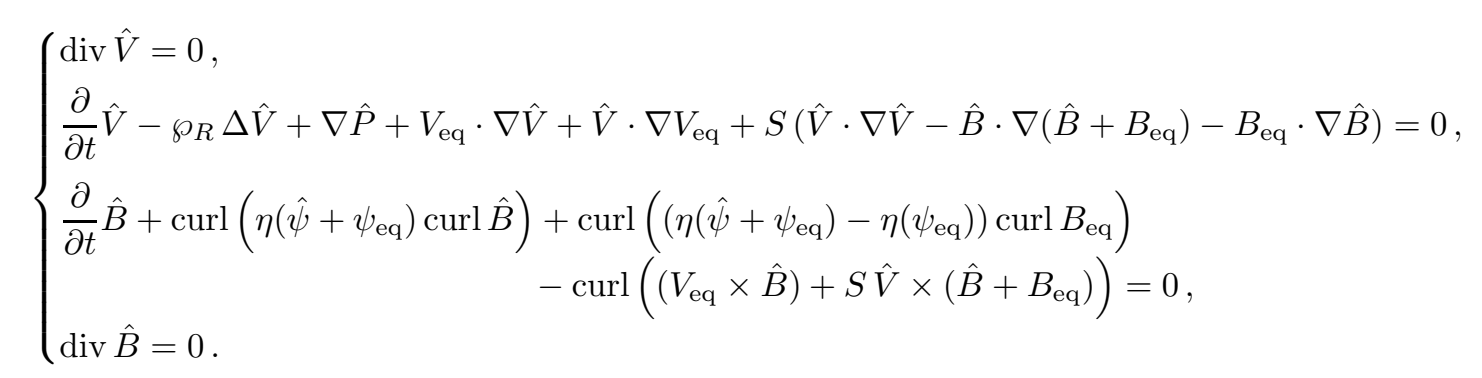

For the unknowns $V$ and $B$ of equations (1), we consider the following boundary conditions at $x= \pm 1 / 2$ :

$V \cdot n=u_{e}, \operatorname{curl} V \times n=g_{e}, B \cdot n=0, \eta(\psi) \operatorname{curl} B \times n=(V \times B) \times n$, and, at $y=0, L: V, \operatorname{curl} V \times$ $n, B, \eta(\psi) \operatorname{curl} B \times n$ periodic.

We also need to impose nonhomogeneous flux conservation conditions by prescribing the numerical values of $\int_{-1 / 2}^{1 / 2} B_{y}(x, 0) \mathrm{d} x$ and $\int_{-1 / 2}^{1 / 2} V_{y}(x, 0) \mathrm{d} x$, where $B_{y}$ and $V_{y}$ are the $y$-components of the magnetic field and of the velocity (such flux conservation conditions allow to define the projection of $B$ and $V$ on the space $H_{c, \text { per }}$, 
generated by the unit vector $e_{y}$ in the $y$ direction, in the next decomposition $(9)$ of $\left(L^{p}(\Omega)\right)^{2}$ into topological supplementary subspaces).

The quantities $S_{e}, u_{e}, g_{e}$ and the flux must be assumed to be compatible (for instance $\int_{\Omega} S_{e} \mathrm{~d} x \mathrm{~d} y=$ $\int_{0}^{L}\left(u_{e}(1 / 2, y)-u_{e}(-1 / 2, y)\right) \mathrm{d} y$. Rather than to specify these data, we assume that the physically given equilibrium quantities $V_{\text {eq }}, B_{\text {eq }}$ (and $\psi_{\text {eq }}$ ), $P_{\text {eq }}$ verify the equations (1) and the boundary conditions (defining then compatible data). As we told above, we are interested in the perturbations $\hat{V}, \hat{B}$ and $\hat{P}$ of the equilibrium, solutions of the equations (2), for which we obtain the following homogeneous boundary conditions at $x= \pm 1 / 2$

$$
\begin{aligned}
& \begin{array}{l}
\hat{V} \cdot n=0, \\
\operatorname{curl} \hat{V} \times n=0, \\
\hat{B} \cdot n=0,
\end{array} \\
&\left(\eta\left(\hat{\psi}+\psi_{\text {eq }}\right) \operatorname{curl} \hat{B}\right) \times n=-\left(\left(\eta\left(\hat{\psi}+\psi_{\text {eq }}\right)-\eta\left(\psi_{\text {eq }}\right)\right) \operatorname{curl} B_{\text {eq }}-V_{\text {eq }} \times \hat{B}\right) \times n+S \hat{V} \times\left(\hat{B}+B_{\text {eq }}\right) \times n,
\end{aligned}
$$

and, at $y=0, L$, the following periodic conditions

$$
\begin{gathered}
\hat{V}, \operatorname{curl} \hat{V} \times n, \hat{B} \text { periodic, } \\
\eta\left(\hat{\psi}+\psi_{\text {eq }}\right) \operatorname{curl} \hat{B} \times n \text { periodic. }
\end{gathered}
$$

We also need the flux conservation condition

$$
\int_{-1 / 2}^{1 / 2} \hat{B}_{y}(x, 0) \mathrm{d} x=0,
$$

and also a flux condition for $\hat{V}$

$$
\int_{-1 / 2}^{1 / 2} \hat{V}_{y}(x, 0) \mathrm{d} x=0
$$

Sometimes, for instance in Theorem 1, we shall introduce a nonhomogeneous condition (8) for the velocity.

Finally, $\hat{V}, \hat{B}$ and $\hat{P}$ are solutions of $(2-8)$.

\section{Steady PROBlem EXPRESSED AS A FUnCTIONAL EQUATION}

The steady problem is obtained by removing from equations (2) terms $\frac{\partial}{\partial t} \hat{V}$ and $\frac{\partial}{\partial t} \hat{B}$.

\subsection{Functional spaces}

Consider $\Omega=]-1 / 2,1 / 2[\times] 0, L\left[\right.$, the Sobolev spaces $W^{m, p}(\Omega)$, with $m$ integer $\geq 1$, and $p \geq 1$, and define $W_{\text {per }}^{m, p}(\Omega)$ as the space of functions of $W^{m, p}(\Omega)$ which are periodic in $y$ :

$$
\begin{aligned}
& W_{\text {per }}^{m, p}(\Omega)=\left\{v \in W^{m, p}(\Omega) ; v(x, 0)=v(x, L), \cdots, \frac{\partial^{m-1}}{\partial y^{m-1}} v(x, 0)=\frac{\partial^{m-1}}{\partial y^{m-1}} v(x, L), \text { a.e. } x \text { in }\right]-1 / 2,1 / 2[\}, \\
& W_{0, \text { per }}^{m, p}(\Omega)=\left\{v \in W_{\text {per }}^{m, p}(\Omega) ; v\left( \pm \frac{1}{2}, y\right)=\cdots, \frac{\partial^{m-1}}{\partial x^{m-1}} v\left( \pm \frac{1}{2}, y\right)=0, \text { a.e. } y \text { in }\right] 0, L[\} .
\end{aligned}
$$


We also define the following spaces

$$
\begin{aligned}
K_{\mathrm{per}}^{p}= & \left\{u \in L^{p}(\Omega)^{2}, \operatorname{div} u=0, u \cdot n=0 \text { at } x= \pm 1 / 2 \text { and } u \cdot n \text { periodic at } y=0, L\right. \\
& \text { with } \left.\int_{-1 / 2}^{1 / 2} u_{y}(x, 0) \mathrm{d} x=0, u_{y} \text { being the } y-\text { component of } u\right\}, \\
H_{c, \text { per }}(\Omega)= & \left\{u \in L^{p}(\Omega)^{2}, \operatorname{div} u=0, \operatorname{curl} u=0, u \cdot n=0 \text { at } x= \pm 1 / 2, u \text { periodic at } y=0, L\right\} .
\end{aligned}
$$

We now consider the following space decomposition into closed supplementary subspaces

$$
L^{p}(\Omega)^{2}=H_{c, \text { per }}(\Omega)+K_{\text {per }}^{p}+\left\{\nabla p, p \in W_{\text {per }}^{1, p}(\Omega)\right\}, \quad 1<p<\infty .
$$

This space decomposition, obtained by Saramito [8] for $p=2$, is obtained for all $1<p<\infty$ in the same way by substituting $L^{2}$ 's scalar product by the dual product between $L^{p}$ and $L^{q}(1 / p+1 / q=1)$. We also have

$$
\begin{aligned}
W_{\text {per }}^{1, p}(\Omega)^{2}= & \left\{u \in L^{p}(\Omega)^{2} ; \operatorname{div} u \in L^{p}(\Omega), \operatorname{curl} u \in L^{p}(\Omega), u \text { periodic in } y, \tilde{u} \cdot n \in W_{\text {loc }}^{1-\frac{1}{p}, p}(\mathbb{R}) \text { at } x= \pm 1 / 2,\right. \\
& \text { where } \tilde{u} \text { is the extension of } u \text { by periodicity in the } y \text { direction }\}
\end{aligned}
$$

$W_{\text {per }}^{1, p}(\Omega)^{2}=\left\{u \in L^{p}(\Omega)^{2} ; \operatorname{div} u \in L^{p}(\Omega), \operatorname{curl} u \in L^{p}(\Omega), u\right.$ periodic in $y, \tilde{u} \times n \in W_{\text {loc }}^{1-\frac{1}{p}, p}(\mathbb{R})$ at $x= \pm 1 / 2$, where $\tilde{u}$ is the extension of $u$ by periodicity in the $y$ direction $\}$.

In [9] (Lem. 2.2, Chap. 2) two analogous equivalent definitions of $W^{1, p}(\mathcal{O})$ are given, $\mathcal{O}$ being some smooth domain. It is a generalization of a well-known result saying that if $u \in\left(L^{2}(\mathcal{O})\right)^{2}$, div $u \in L^{2}(\mathcal{O}), \operatorname{curl} u \in L^{2}(\mathcal{O})$, with either $u \cdot n=0$ on $\partial \mathcal{O}$ or $u \times n=0$ on $\partial \mathcal{O}$, then $u \in H^{1}(\mathcal{O})$. The decompositions $(10,11)$ are easily obtained, applying results of Saramito [9] on a smooth domain containing $\Omega$, to the extension by periodicity $\tilde{u}$, after multiplication by a truncation function in the $y$ direction. We also define

$$
\begin{aligned}
X_{\mathrm{per}}^{p}= & \left\{u \in L^{p}(\Omega)^{2} ; \operatorname{div} u \in L^{p}(\Omega), \operatorname{curl} u \in L^{p}(\Omega), u \text { periodic at } y=0, L,\right. \\
& \left.u \cdot n=0 \text { at } x= \pm 1 / 2, \text { projection of } u \text { on } H_{c, \text { per }}(\Omega), \text { defined by }(9), \text { vanishes }\right\}
\end{aligned}
$$

We note that each u of $X_{\text {per }}^{p}$ belongs to $W_{\text {per }}^{1, p}(\Omega)^{2}$.

We have as in [8]:

Lemma 1. For $1<p<\infty$

$$
\|\| u \|_{p}=\left(\int_{\Omega}|\operatorname{curl} u|^{p} \mathrm{~d} \Omega+\int_{\Omega}|\operatorname{div} u|^{p} \mathrm{~d} \Omega\right)^{\frac{1}{p}}
$$

is a norm in $X_{\mathrm{per}}^{p}$ equivalent to that of $\left(W^{1, p}(\Omega)\right)^{2}$.

\subsection{Coerciveness lemma}

Lemma 2. Let $a \in L^{\infty}(\Omega)$ such that there exists $\mu \in \mathbb{R}_{+}^{*}$ with $0<\mu \leq a(x)$ a.e. in $\Omega$. There exists a constant $C$, depending of $\mu$, such that, for all $u \in X_{\mathrm{per}}^{p}$, with $1<p<\infty$, we have

$$
\|u \mid\|_{p} \leq C \sup _{\substack{v \in X_{\text {per }}^{q} \\\|v\|_{q} \leq 1}} \int_{\Omega} a(x)[\operatorname{curl} u \operatorname{curl} v+\operatorname{div} u \operatorname{div} v] \mathrm{d} \Omega .
$$


The proof of this lemma will be done in two steps. We first obtain the announced result for the case $a=$ const. $(a(x)=1)$ and in a second step we prove the result in the general case.

\section{Proof.}

Step 1. $a(x)=1$

(i) For $h \in L^{q}(\Omega) / \mathbb{R}$, there exists one and only one $\phi \in W_{\text {per }}^{2, q}(\Omega) / \mathbb{R}$ such that $\Delta \phi=h$ in $\Omega, \nabla \phi \cdot n=0$ at $x= \pm 1 / 2 ; \phi$ and $\nabla \phi \cdot n$ periodic at $y=0, L ; \int_{\Omega} \phi \mathrm{d} \Omega=0$.

So $v=\nabla \phi$ belongs to $X_{\text {per }}^{q}$ and one has for all $\mathrm{u} \in X_{\text {per }}^{p}$

and then

$$
\begin{gathered}
\|\operatorname{div} u\|_{p}=\sup _{h \in L^{q}(\Omega) / \mathbb{R},\|h\|_{q} \leq 1} \int_{\Omega} \operatorname{div} u h \mathrm{~d} \Omega, \\
\|\operatorname{div} u\|_{p} \leq \sup _{\substack{v \in X_{\text {per }}^{q}, v=\nabla \phi \\
\|\operatorname{div}\|_{q} \leq 1 ;}} \int_{\Omega} \operatorname{div} u \operatorname{div} v \mathrm{~d} \Omega,
\end{gathered}
$$

$$
\|\operatorname{div} u\|_{p} \leq \sup _{\substack{v \in X_{\text {per }}^{q}, \operatorname{curl} v=0,\|\operatorname{div} v\|_{q} \leq 1}} \int_{\Omega} \operatorname{div} u \operatorname{div} v \mathrm{~d} \Omega .
$$

(ii) For $h \in L^{q}(\Omega)$, there exists $v \in W_{\operatorname{per}}^{1, q}(\Omega)^{2}$ such that $h=\operatorname{curl} v(v=\operatorname{curl} \phi$, where $\phi$ is a solution of equation $-\Delta \phi=h$ is an example of $v$ which verifies relation $h=\operatorname{curl} v)$, so

$$
\begin{aligned}
\|\operatorname{curl} u\|_{p} & =\sup _{h \in L^{q}(\Omega),\|h\|_{q} \leq 1} \int_{\Omega} \operatorname{curl} u h \mathrm{~d} \Omega \\
& \leq \sup \underset{\substack{v \in W_{\mathrm{p}}^{1, q}(\Omega)^{2} \\
\|\operatorname{curl} v\|_{q} \leq 1}}{2} \int_{\Omega} \operatorname{curl} u \operatorname{curl} v \mathrm{~d} \Omega .
\end{aligned}
$$

By extension of a result due to Foias and Temam [4] and to Saramito [8] to spatial periodic case, one has that curl is an isomorphism from $W_{\text {per }}^{1, q}(\Omega)^{2} \cap K_{\text {per }}^{q}$ to the space $\operatorname{curl}\left(W_{\text {per }}^{1, q}(\Omega)^{2}\right) \subset L^{q}(\Omega)$, so

$$
\|\operatorname{curl} u\|_{p} \leq \sup _{\substack{X_{\text {per }}^{q}, \operatorname{div} v=0 \\\|\operatorname{curl} v\|_{q} \leq 1}} \int_{\Omega} \operatorname{curl} u \operatorname{curl} v \mathrm{~d} \Omega .
$$

Adding (i) and (ii) one gets the expected result for the case $a(x)=$ constant.

Step 2. $a$ is a positive bounded function

We can choose without restriction the function $a$ of the form

$$
a(x)=1+\epsilon(x), \text { with }|\epsilon(x)| \leq \epsilon_{1}<1 .
$$

For this choice of $a$, and applying Hölder's inequality, one has

$$
\int_{\Omega} a \operatorname{curl} u \operatorname{curl} v \mathrm{~d} \Omega=\int_{\Omega}(1+\epsilon) \operatorname{curl} u \operatorname{curl} v \mathrm{~d} \Omega \geq \int_{\Omega} \operatorname{curl} u \operatorname{curl} v \mathrm{~d} \Omega-\epsilon_{1}\|\operatorname{curl} u\|_{p}\|\operatorname{curl} v\|_{q} .
$$

Then, $\forall v \in X_{\text {per }}^{q}$ with div $\mathrm{v}=0$ and $\|\operatorname{curl} v\|_{q} \leq 1$, one has

$$
\int_{\Omega} a \operatorname{curl} u \operatorname{curl} v \mathrm{~d} \Omega \geq \int_{\Omega} \operatorname{curl} u \operatorname{curl} v \mathrm{~d} \Omega-\epsilon_{1}\|\operatorname{curl} u\|_{p} .
$$


Taking into account part (ii) of the first step, we have

$$
\left(1-\epsilon_{1}\right)\|\operatorname{curl} u\|_{p} \leq \sup _{\substack{v \in X_{\text {per }}^{q}, \operatorname{div} v=0 \\\|\operatorname{curl} v\|_{q} \leq 1}} \int_{\Omega} a \operatorname{curl} u \operatorname{curl} v \mathrm{~d} \Omega .
$$

As in the first step, we obtain another inequality which, added to the preceding one, proves the lemma.

\subsection{Resolution of an auxiliary problem}

Proposition 1. Let $a \in L^{\infty}(\Omega), \mu \in \mathbb{R}_{+}^{*}$ such that $0<\mu \leq a(x)$ a. e. in $\Omega$. Let $1<p<\infty$, and $f$ of the form:

$$
f=\operatorname{curl} g \text {, with } g \in L^{p}(\Omega) .
$$

Then there exists one and only one $v \in W_{\text {per }}^{1, p}(\Omega)^{2}$ that verifies the following equations in a weak sense

$$
\left\{\begin{array}{l}
\operatorname{div} v=0 \\
\operatorname{curl}(a \operatorname{curl} v)=f \text { in }\left(\mathcal{D}^{\prime}(\Omega)\right)^{2} \\
v \cdot n=0 \text { and }(a \operatorname{curl} v-g) \times n=0 \text { at } x= \pm 1 / 2 \\
v \text { and }(\operatorname{a} \operatorname{curl} v-g) \times n \text { periodic in } y=0, L \\
\int_{-1 / 2}^{1 / 2} v_{y}(x, 0) \mathrm{d} x=0(c f .(8))
\end{array}\right.
$$

and there exists a constant $C$ depending only on $\Omega$ and $\mu$ such that

$$
\|v\|_{W_{\text {per }}^{1, p}(\Omega)} \leq C\|g\|_{p} .
$$

Moreover, if $g \in W_{\text {per }}^{1, p}(\Omega)$ and $a \in W_{\text {per }}^{1, \infty}(\Omega)$, then $v \in W_{\text {per }}^{2, p}(\Omega)$.

Proof.

(i) Existence of solution:

For $g \in L^{p}(\Omega)$, we deduce from Lemma 2 and Theorem 3.1 of Chapter 6 of [6], that there exists a unique $v \in X_{\mathrm{per}}^{p}$ solution of the variational problem

$$
\int_{\Omega} a \operatorname{curl} v \operatorname{curl} w \mathrm{~d} \Omega+\int_{\Omega} a \operatorname{div} v \operatorname{div} w \mathrm{~d} \Omega=\int_{\Omega} g \cdot \operatorname{curl} w \mathrm{~d} \Omega \text { for all } w \in X_{\mathrm{per}}^{q}
$$

Relation (15) is also true for all $w \in H_{c, \text { per }}(\Omega)$; so it is true for all $w \in W_{\text {per }}^{1, q}(\Omega)^{2}$ which verify relation $w \cdot n=0$ at $x= \pm 1 / 2$; we then deduce from (15) that $v$ verifies

$$
\operatorname{curl}(a \operatorname{curl} v)-\nabla(a \nabla \cdot v)=f \text { in } \mathcal{D}^{\prime}(\Omega)^{2} .
$$

Due to the fact that $v \in X_{\text {per }}^{p}$ we also have $v \cdot n=0$ at $x= \pm 1 / 2$ and $v$ periodic in $y$.

(ii) Let us show that if $\operatorname{div} f=0$ then $\operatorname{div} v=0$.

Let $\psi \in L^{q}(\Omega) / \mathbb{R}$. The map $\psi \longmapsto \phi$ defined by

$$
\begin{aligned}
& \Delta \phi=\psi ; \frac{\partial}{\partial n} \phi=0 \text { en } x= \pm \frac{1}{2} ; \\
& \phi, \frac{\partial}{\partial n} \phi \text { periodic in } y
\end{aligned}
$$


is an isomorphism from $L^{q}(\Omega) / \mathbb{R}$ on $\left\{\phi \in W_{\text {per }}^{2, q}(\Omega), \frac{\partial}{\partial n} \phi=0\right.$ at $\left.x= \pm 1 / 2, \int_{\Omega} \phi=0\right\}$.

For such a $\phi, w=\nabla \phi$ belongs to $X_{\mathrm{per}}^{q}$ and for this $w(15)$ becomes

$$
\int_{\Omega} a \Delta \phi \operatorname{div} v \mathrm{~d} \Omega=0
$$

i.e.

$$
\int_{\Omega} a \operatorname{div} v \psi \mathrm{d} \Omega=0 \text { for all } \psi \in L^{q}(\Omega) / \mathbb{R}
$$

which gives $\operatorname{div} v=0$ (for any $\psi_{1} \in L^{q}(\Omega), \exists$ a constant $c$ and $\psi \in L^{q}(\Omega) / \mathbb{R}$ such that $a \psi+c=\psi_{1}$ a.e. in $\Omega$ ).

(iii) Boundary conditions.

Because $v \in X_{\text {per }}^{p}$, the projection of $v$ on $H_{c, \text { per }}(\Omega)$ vanishes, which is equivalent to $\int_{\Omega} v \cdot e_{y} \mathrm{~d} \Omega=0, e_{y}$ being the generator of $H_{c, \text { per }}(\Omega)$. As $\operatorname{div} v=0$, this condition can be written as $\int_{-1 / 2}^{1 / 2} v_{y}(x, 0) \mathrm{d} x=0$, and equation (16) can be written

$$
\operatorname{curl}(a \operatorname{curl} v)=\operatorname{curl} g \text {. }
$$

For $a \in L^{\infty}(\Omega), g \in L^{p}(\Omega)$, if $v \in X_{\mathrm{per}}^{p}$, then $u=\operatorname{curl} v \in L^{p}(\Omega)$ and $a u-g \in L^{p}(\Omega)$. We know from (17) that curl $(a u-g) \in L^{p}(\Omega)$; so the trace of $(a u-g) \times n$ gets meaning in $W^{-\frac{1}{p}, p}(\Gamma)$.

Green's formula applied to (17) gives

$$
\int_{\Omega} a \operatorname{curl} v \operatorname{curl} w \mathrm{~d} \Omega+\int_{\Gamma}(a \operatorname{curl} v-g) \times n \cdot w \mathrm{~d} \Gamma=\int_{\Omega} g \cdot \operatorname{curl} w \mathrm{~d} \Omega,
$$

for all $w \in W_{\text {per }}^{1, q}(\Omega)$.

Taking into account (15) which is true for all $w \in W_{\text {per }}^{1, q}(\Omega)^{2}$ such that $w \cdot n=0$ at $x= \pm 1 / 2$ we have

$$
\int_{\Gamma}(a \operatorname{curl} v-g) \times n \cdot w \mathrm{~d} \Gamma=0
$$

for all $w \in W_{\text {per }}^{1, q^{2}}$ such that $w \cdot n=0$ at $x= \pm 1 / 2$ and this gives boundary conditions for $\operatorname{curl} v \times n$.

(iv) Regularity of the solution: $v \in W_{\text {per }}^{2, p}(\Omega)$.

For $a \in W_{\text {per }}^{1, \infty}(\Omega), g \in W_{\text {per }}^{1, p}(\Omega)$, and $v \in X_{\text {per }}^{p}$, the equation $\operatorname{curl}(a \operatorname{curl} v)=f$ can also be written

$$
-a \Delta v+\nabla a \times \operatorname{curl} v=f \in\left(L^{p}(\Omega)\right)^{2} .
$$

We have shown that $v \in X_{\text {per }}^{p}$, so $\operatorname{curl} v \in L^{p}(\Omega)$ and then $\nabla a \times \operatorname{curl} v \in L^{p}(\Omega)^{2}$. We then deduce from (18) that $v$ also verifies equations

$$
-\Delta v=\frac{1}{a}(f-\nabla a \times \operatorname{curl} v) \in L^{p}(\Omega)^{2} .
$$

In these expressions, we use products of $W^{-1, p}(\Omega)$ by $W^{1, \infty}(\Omega)$.

Regularity $W_{\text {per }}^{2, p}(\Omega)$ of $v$ can then be given by the regularity of Laplace operator for Dirichlet and Neumann boundary conditions.

As usual for second order operators, regularity can also be proved using (15), extensions of $a, v, w$ and $g$ by periodicity, translations in the direction parallel to $e_{y}$, equation (18) for regularity of derivatives $\partial^{2} / \partial x^{2}$, and finally boundary conditions obtained previously in a weak sense. 


\subsection{The stationary problem expressed in a functional equation form}

We are now going to write the steady problem in the form $(I-T(S,)) U=$.0 , where $T(S,$.$) is a compact$ operator in a suitable functional space.

We define

$$
V_{p}=\left\{v \in\left(W_{\text {per }}^{1, p}(\Omega)\right)^{2}, \operatorname{div} v=0, v \cdot n=0 \text { at } x= \pm 1 / 2\right\} .
$$

In the following, we suppose that $\eta \in W^{2, \infty}(\mathbb{R}) \cap C^{2}(\mathbb{R})$ and that there exists a positive real $\mu$ such that $0<\mu \leq \eta(x)$ for all $x \in \mathbb{R}$.

Theorem 1. For all $U=(V, B) \in V_{p}^{2}, p>2$, with $\int_{-1 / 2}^{1 / 2} B_{y}(x, 0) \mathrm{d} x=0$, there exits one and only one $U^{*}=\left(V^{*}, B^{*}\right) \in V_{p}^{2}$, noted $U^{*}=T(S, U)$, that verifies the following equations in a weak sense

$$
\left\{\begin{array}{l}
\wp_{R} \operatorname{curl} \operatorname{curl} V^{*}+\nabla P^{*}+V^{*}=V-V_{\mathrm{eq}} \cdot \nabla V-V \cdot \nabla V_{\mathrm{eq}}-S\left(V \cdot \nabla V-B \cdot \nabla\left(B+B_{\mathrm{eq}}\right)-B_{\mathrm{eq}} \cdot \nabla B\right), \\
\operatorname{curl}\left(\eta\left(\psi+\psi_{\mathrm{eq}}\right) \operatorname{curl} B^{*}\right)=\operatorname{curl}\left(V_{\mathrm{eq}} \times B+S V \times\left(B+B_{\mathrm{eq}}\right)\right)-\operatorname{curl}\left(\left(\eta\left(\psi+\psi_{\mathrm{eq}}\right)-\eta\left(\psi_{\mathrm{eq}}\right)\right) \operatorname{curl} B_{\mathrm{eq}}\right), \\
\operatorname{div} V^{*}=0 \text { and } \operatorname{div} B^{*}=0,
\end{array}\right.
$$

boundary conditions $(3,5,6)$, for $V^{*}$ and $B^{*}$, and at $x= \pm 1 / 2$,

$$
\left(\eta\left(\psi+\psi_{\text {eq }}\right) \operatorname{curl} B^{*}\right) \times n=\left(V_{\text {eq }} \times B+S V \times\left(B+B_{\text {eq }}\right)\right) \times n-\left(\left(\eta\left(\psi+\psi_{\text {eq }}\right)-\eta\left(\psi_{\text {eq }}\right)\right) \operatorname{curl} B_{\text {eq }}\right) \times n,
$$

flux conservation condition (7) for $B_{y}^{*}$, and a nonhomogeneous flux conservation relation for $V^{*}$.

Moreover, if $V_{\mathrm{eq}}=0$, we can remove zero order derivation terms $V^{*}$ and $V$ from $V^{*}$ 's equation and we can add the condition (8) : $\int_{-1 / 2}^{1 / 2} V_{y}^{*}(x, 0) \mathrm{d} x=0$.

The so defined operator $T$ is compact from $V_{p}^{2}$ to $V_{p}^{2}$.

We have, with $C$ a positive constant

$$
\left\|U^{*}\right\|_{V_{p}^{2}} \leq C\|U\|_{V_{p}^{2}}\left(1+\|U\|_{V_{p}^{2}}\right) .
$$

Proof.

(i) Existence and regularity of the magnetic field $B^{*}$ :

Existence and regularity of $B^{*}$ is given by Proposition 1 :

the right-hand side of $B^{*}$ 's equation is of the form $g_{3}=\operatorname{curl} g_{2}$ with

$$
g_{2}=V_{\mathrm{eq}} \times B+S V \times\left(B+B_{\mathrm{eq}}\right)-\left(\eta\left(\psi+\psi_{\mathrm{eq}}\right)-\eta\left(\psi_{\mathrm{eq}}\right)\right) \operatorname{curl} B_{\mathrm{eq}} .
$$

For $p>2, g_{2} \in W_{\text {per }}^{1, p}(\Omega)$, then $g_{3}$ verifies the hypothesis done on $f$ in Proposition 1.

For a given $B \in V_{p}$ there exists one and only one $\psi$ in $W_{\text {per }}^{2, p}(\Omega)$ solution of the equations

$$
\left\{\begin{array}{l}
-\Delta \psi=-\operatorname{curl} B \\
\psi=0 \text { in } x= \pm \frac{1}{2} \\
\psi, \frac{\partial}{\partial y} \psi \text { periodic in } y=0, L
\end{array}\right.
$$

For $B \in V_{p}$ with flux condition (7), this is equivalent to $B=e_{z} \times \nabla \psi$.

With $p>2, \psi \in W_{\text {per }}^{2, p}(\Omega) \subset W_{\text {per }}^{1, \infty}(\Omega)$, and with a result of Simon [11], we conclude that $\eta\left(\psi+\psi_{\text {eq }}\right) \in$ $W_{\text {per }}^{1, \infty}(\Omega)$. 
Then we can apply proposition $\mathrm{P} 1$, and we obtain existence of an unique $B^{*} \in W_{\text {per }}^{2, p}(\Omega)$.

(ii) Existence and regularity of the velocity $V^{*}$ :

Existence and regularity of an unique $V^{*} \in W_{\text {per }}^{2, p}(\Omega)$ which verifies $V^{*}$ 's equations is obtained by extension to periodic case of a result due to Saramito [8].

More precisely, using (9), proving a proposition analogous to Proposition 1 (with $a=1$ and $\int_{\Omega} f$.w in the r.h.s. of (15), for $f \in K_{\text {per }}^{p}$, and using Fredholm alternative, we obtain easily the results for $V^{*}$.

Compactness of $T$ results from compact imbeding of $W_{\mathrm{per}}^{2, p}(\Omega)$ into $W_{\text {per }}^{1, p}(\Omega)$.

Corollary 1. The stationary problem deduced from (2-8) is then reduced to find $U \in V_{p}^{2}$ such that

$$
U-T(S, U)=0
$$

where $T$ is the compact operator defined in Theorem 1.

We now introduce a linearized operator, noted $A$, in a neighbourhood of zero.

Proposition 2. For all $U=(V, B) \in V_{p}^{2}, 1<p<\infty$, with $\int_{-1 / 2}^{1 / 2} B_{y}(x, 0) \mathrm{d} x=0$, there exists a unique $\bar{U}=(\bar{V}, \bar{B}) \in V_{p}^{2}$, noted $\bar{U}=A(S, U)$, such that, in a weak sense

$$
\left\{\begin{array}{l}
\operatorname{div} \bar{V}=0, \\
\wp_{R} \operatorname{curl} \operatorname{curl} \bar{V}+\nabla \bar{P}+\bar{V}=V-V_{\text {eq }} \cdot \nabla V-V \cdot \nabla V_{\text {eq }}+S\left(B \cdot \nabla B_{\text {eq }}+B_{\text {eq }} \cdot \nabla B\right), \\
\operatorname{curl}\left(\eta\left(\psi_{\text {eq }}\right) \operatorname{curl} \bar{B}\right)=\operatorname{curl}\left(V_{\text {eq }} \times B+S V \times B_{\text {eq }}\right)-\operatorname{curl}\left(\eta^{\prime}\left(\psi_{\text {eq }}\right) \psi \operatorname{curl} B_{\text {eq }}\right), \\
\operatorname{div} \bar{B}=0,
\end{array}\right.
$$

and $\bar{U}$ also verifies boundary conditions $(3,5), \operatorname{curl} \bar{B} \times n$ periodic at $y=0, L$ and

$$
\left(\eta\left(\psi_{\mathrm{eq}}\right) \operatorname{curl} \bar{B}\right) \times n=\left[V_{\mathrm{eq}} \times B+S V \times B_{\mathrm{eq}}-\eta^{\prime}\left(\psi_{\mathrm{eq}}\right) \psi \operatorname{curl} B_{\mathrm{eq}}\right] \times n \text { at } x= \pm \frac{1}{2}
$$

the flux conservation condition (7) for $\bar{B}_{y}$, and a flux conservation relation for $\bar{V}$.

Moreover, if $V_{\mathrm{eq}}=0$, we can remove zero order derivation terms $\bar{V}$ and $V$ from $\bar{V}$ 's equation and we can add the condition (7) for $\bar{V}_{y}$.

The so defined operator $A$ is a compact operator from $V_{p}^{2}$ to $V_{p}^{2}$.

Proof. The results of this proposition are obtained in the same way as those of Theorem 1.

Proposition 3. Consider $\eta \in W^{2, \infty}(\mathbb{R}) \cap C^{2}(\mathbb{R})$, and $p>2$, then $A$ is the differential operator of $T$ with respect to $U$ at $(S, 0)$.

Proof. To obtain the result announced in this proposition, we have to show that

$$
\frac{\|T(S, U)-T(S, 0)-A(S, U)\|_{V_{p}^{2}}}{\|U\|_{V_{p}^{2}}} \longrightarrow 0 \text { as }\|U\|_{V_{p}^{2}} \longrightarrow 0 \text {. }
$$

For so doing let $\tilde{U}=U^{*}-\bar{U}=(\tilde{V}, \tilde{B})$, where $U^{*}=T(S, U)$ and $\bar{U}=A(S, U)$, with $T(S, 0)=0$. 
Due to Theorem 1 and Proposition 2, $\tilde{U}$ verifies

$$
\left\{\begin{array}{l}
\operatorname{div} \tilde{V}=0, \\
\wp_{R} \operatorname{curl} \operatorname{curl} \tilde{V}+\nabla \tilde{P}+\tilde{V}=-S(V \cdot \nabla V-B \cdot \nabla B), \\
\operatorname{curl}\left(\eta\left(\psi_{\mathrm{eq}}\right) \operatorname{curl} \tilde{B}\right)=S \operatorname{curl}(V \times B)-\operatorname{curl}\left(\left(\eta\left(\psi+\psi_{\mathrm{eq}}\right)-\eta\left(\psi_{\mathrm{eq}}\right)\right) \operatorname{curl} B^{*}\right) \\
\quad-\operatorname{curl}\left(\left(\eta\left(\psi+\psi_{\mathrm{eq}}\right)-\eta\left(\psi_{\mathrm{eq}}\right)-\eta^{\prime}\left(\psi_{\mathrm{eq}}\right) \psi\right) \operatorname{curl} B_{\mathrm{eq}}\right), \\
\operatorname{div} \tilde{B}=0,
\end{array}\right.
$$

boundary conditions $(3,5), \operatorname{curl} \tilde{B} \times n$ periodic, the flux conservation relation $(7)$, a flux conservation relation for $\tilde{V}$, and, at $x= \pm 1 / 2$, the condition

$$
\begin{aligned}
\eta\left(\psi_{\text {eq }}\right) \operatorname{curl} \tilde{B} \times n= & \left(S V \times B-\left(\eta\left(\psi+\psi_{\text {eq }}\right)-\eta\left(\psi_{\text {eq }}\right)\right) \operatorname{curl} B^{*}\right) \times n \\
& -\left(\left(\eta\left(\psi+\psi_{\text {eq }}\right)-\eta\left(\psi_{\text {eq }}\right)-\eta^{\prime}\left(\psi_{\text {eq }}\right) \psi\right) \operatorname{curl} B_{\text {eq }}\right) \times n .
\end{aligned}
$$

From these equations we deduce the following estimates for the equation of $\tilde{B}$ (using $\|\psi\|_{2, p} \leq C\|B\|_{1, p}$ as in the proof of Th. 1):

(i)

(ii)

$$
\begin{aligned}
\left\|\left(\eta\left(\psi+\psi_{\mathrm{eq}}\right)-\eta\left(\psi_{\mathrm{eq}}\right)\right) \operatorname{curl} B^{*}\right\|_{p} & \leq\left\|\eta\left(\psi+\psi_{\mathrm{eq}}\right)-\eta\left(\psi_{\mathrm{eq}}\right)\right\|_{\infty}\left\|\operatorname{curl} B^{*}\right\|_{p} \\
& \leq C\|\eta\|_{1, \infty}\|\psi\|_{\infty}\left\|B^{*}\right\|_{1, p} \\
& \leq C\|\psi\|_{1, p}\left\|B^{*}\right\|_{1, p} \\
& \leq C\|U\|_{V_{p}^{2}}\|U\|_{V_{p}^{2}}\left(1+\|U\|_{V_{p}^{2}}\right) .
\end{aligned}
$$

$$
\begin{gathered}
\left\|\left(\eta\left(\psi+\psi_{\mathrm{eq}}\right)-\eta\left(\psi_{\mathrm{eq}}\right)-\eta^{\prime}\left(\psi_{\mathrm{eq}}\right) \psi\right) \operatorname{curl} B_{\mathrm{eq}}\right\|_{p} \leq C\|\eta\|_{2, \infty}\|\psi\|_{\infty}^{2}\left\|\operatorname{curl} B_{\mathrm{eq}}\right\|_{p} \\
\leq C\|U\|_{V_{p}^{2}}^{2} .
\end{gathered}
$$

The other estimates are easily obtained, for the other terms of the two right-hand sides of the equations of $\tilde{B}$ and $\tilde{V}$. From these estimates, one deduces for $\tilde{U}$ (see the estimate in Prop. 1)

$$
\|\tilde{U}\|_{V_{p}^{2}} \leq C\|U\|_{V_{p}^{2}}^{2}\left(\|U\|_{V_{p}^{2}}+1\right)
$$

\subsection{Bifurcated solutions for the stationary problem}

Let us define $F(S, U)=U-T(S, U)$, where $T$ is the operator defined in Theorem 1 .

The steady problem can then be written

$$
F(S, U)=0 \text { for } U \in V_{p}^{2}, S \in \mathbb{R} .
$$

We see that $U=0$ is a trivial solution of the problem; $F(S, U)=0$ for all $S \in \mathbb{R}$.

We can now apply a theorem due to Crandall and Rabinowitz [3] to our problem, to show the existence of bifurcated solutions from the trivial solution.

Theorem 2. Let us assume that:

(a) the partial derivatives $F_{S}, F_{U}$, and $F_{S, U}$ exist and are continuous (this is true for the studied problem, see [1])

(b) The kernel of $F_{U}\left(S_{0}, 0\right)$ is of dimension 1 , spanned by $U_{0}$, for a value $S_{0}$ of the bifurcation parameter $S$,

(c) $F_{S, U}\left(S_{0}, 0\right) U_{0} \notin$ Range $\left(F_{U}\left(S_{0}, 0\right)\right)$. 
Then a branch of steady solutions bifurcates from the trivial branch $(S, 0)$ at $\left(S_{0}, 0\right)$, in the space $\mathbb{R} \times V_{p}^{2}$.

Once we have replaced the problem (2-8) by the functional equation $F(S, U)=0$, for $S \in \mathbb{R}$ and $U=(\hat{V}, \hat{B}) \in$ $V_{p}^{2}$, Theorem 2 tells us that, in a neighbourhood of $\left(S_{0}, 0\right)$ in the space $\mathbb{R} \times V_{p}^{2}$, we have two branches of non linear solutions crossing at the point $\left(S_{0}, 0\right)$ : the trivial branch $(S, 0)$ and the new bifurcated branch which, as can be shown in the proof of the Theorem 2, can be represented, using a real parameter $t$ in a neighbourhood of zero, as $(S(t), U(t))$, with $S(t) \sim S_{0}$ and $U(t) \sim t U_{0}$ when t tends to zero. For the problem with unknowns $(V, B)$ (see Sect. 1), it means that a new non linear branch bifurcates at $\left(S_{0},\left(V_{\text {eq }}, B_{\text {eq }}\right)\right)$ from the equilibrium branch $\left(S,\left(V_{\text {eq }}, B_{\text {eq }}\right)\right)$ to the branch $\left(S(t),\left(V_{\text {eq }}+S(t) \hat{V}(t), B_{\text {eq }}+\hat{B}(t)\right)\right)$, with $t$ in a neighbourhood of zero as above. Then, due to Theorem 2, it is enough to solve a linear problem (i.e. to find the kernel of $F_{U}\left(S_{0}, 0\right)$ ) to conclude that the full non linear system of equations possesses new solutions. In Section 4, considering a physically given equilibrium such that the field lines of the magnetic field are all parallel to the unit vector in the $y$ direction $e_{y}$, we shall numerically solve that linear problem to obtain quantities $S_{0}$ and $U_{0}$. Looking now to the field lines of the bifurcated magnetic field $B_{\text {eq }}+\hat{B}(t)$ we could see that these lines are no longer parallel, but that they lead to the formation of a magnetic island, leading then to a "tearing" of the previous equilibrium parallel field lines configuration.

In order to apply Theorem 2 we are now going to study the kernel of the linearized operator $F_{U}\left(S_{0}, 0\right)$. This study will be done numerically.

\section{Numerical Resolution of the Linearized STEADY PROBlem}

We want to study the kernel of the linearized operator $F_{U}\left(S_{0}, 0\right)=I_{d}-A\left(S_{0}, 0\right)$. For so doing, we consider the following problem, where unknowns are approximated by finite differences.

Taking into account the choice of an equilibrium depending only on $x$, periodic boundary conditions and symmetry properties of the solutions $(c f .[8])$, every vector $(V, B, P)$ of the kernel can be written as a Fourier' series in $y$ of general term

$$
\begin{array}{lll}
V_{x}=v_{x}(x) \cos (m k y) & V_{y}=v_{y}(x) \sin (m k y) & P=p(x) \cos (m k y) \\
B_{x}=b_{x}(x) \sin (m k y) & B_{y}=b_{y}(x) \cos (m k y)
\end{array}
$$

where $k=2 \pi / L$, and $m$ is a positive integer.

Using divergence equation one gets for $b_{x}(x), v_{x}(x)$ and $p(x)$ the following equations in which we write $\mathrm{b}, v$ for $b_{x}$ and $v_{x}$

$$
\left\{\begin{array}{l}
-\wp_{R} \Delta v+\frac{\mathrm{d}}{\mathrm{d} x}\left(u_{0} v\right)+\frac{\mathrm{d}}{\mathrm{d} x} p=S \times(m k \theta b) \\
\Delta p+2 \frac{\mathrm{d}}{\mathrm{d} x}\left(v \frac{\mathrm{d}}{\mathrm{d} x} u_{0}\right)-v \frac{\mathrm{d}^{2}}{\mathrm{~d} x^{2}} u_{0}=S \times\left(2 m k b \frac{\mathrm{d}}{\mathrm{d} x} \theta\right) \\
-\frac{\mathrm{d}}{\mathrm{d} x}\left(\eta\left(\psi_{\text {eq }}\right) \frac{\mathrm{d}}{\mathrm{d} x} b\right)+\left(\eta^{\prime}\left(\psi_{\text {eq }}\right) \theta+u_{0}\right) \frac{\mathrm{d}}{\mathrm{d} x} b+\left(m^{2} k^{2} \eta\left(\psi_{\text {eq }}\right)-\eta^{\prime}\left(\psi_{\text {eq }}\right) \frac{\mathrm{d}}{\mathrm{d} x} \theta\right) b=S \times(-m k \theta v)
\end{array}\right.
$$

where

$$
\Delta=\frac{\mathrm{d}^{2}}{\mathrm{~d} x^{2}}-m^{2} k^{2} \quad, \quad \theta=\frac{\mathrm{d}}{\mathrm{d} x} \psi_{\text {eq }}, \quad u_{0}=\left(V_{\mathrm{eq}}\right)_{x} .
$$

The boundary conditions at $x= \pm 1 / 2$ are

$$
v=b=0, \frac{\mathrm{d}}{\mathrm{d} x}\left(u_{0} v\right)+\frac{\mathrm{d}}{\mathrm{d} x} p=0 .
$$

After discretization by finite difference method, previous equations can be written in the form $A X=S B X$. 


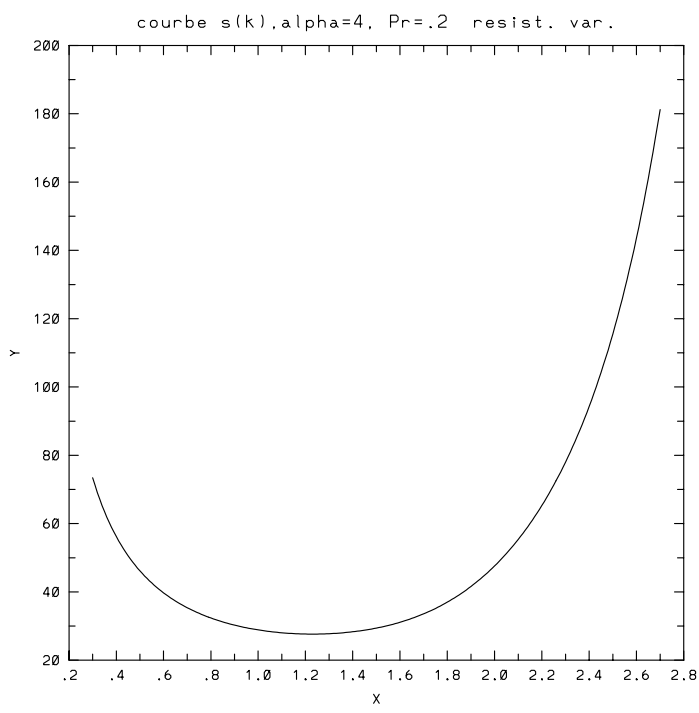

Figure 1. Curve $\mathrm{S}(\mathrm{k})$.

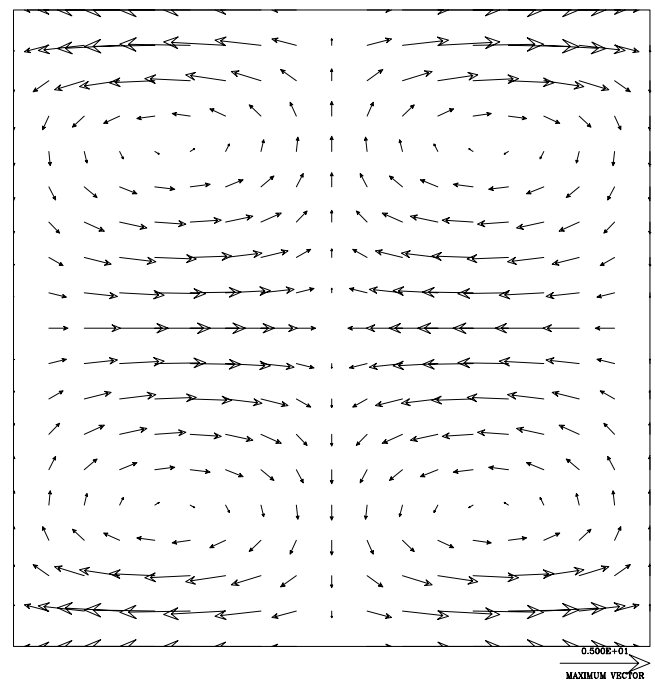

FIgURE 2. Velocity.

We then solve this spectral problem by inverse iteration to get for $\wp_{R}, m$, and $k$ fixed the smallest value of $S$ for which the kernel of the discretized linearized operator is not trivial.

\section{DESCRIPTION OF THE NUMERICAL RESUltS}

To obtain these results, we consider a resistivity which depends on the equilibrium flux function

$$
\eta\left(\psi_{\mathrm{eq}}\right)=\frac{2 \alpha}{\alpha+\sinh (\alpha)} \exp \left(2 \alpha \psi_{\mathrm{eq}}\right)=\frac{2 \alpha}{\alpha+\sinh (\alpha)} \cosh ^{2}(\alpha x), \alpha \neq 0 .
$$




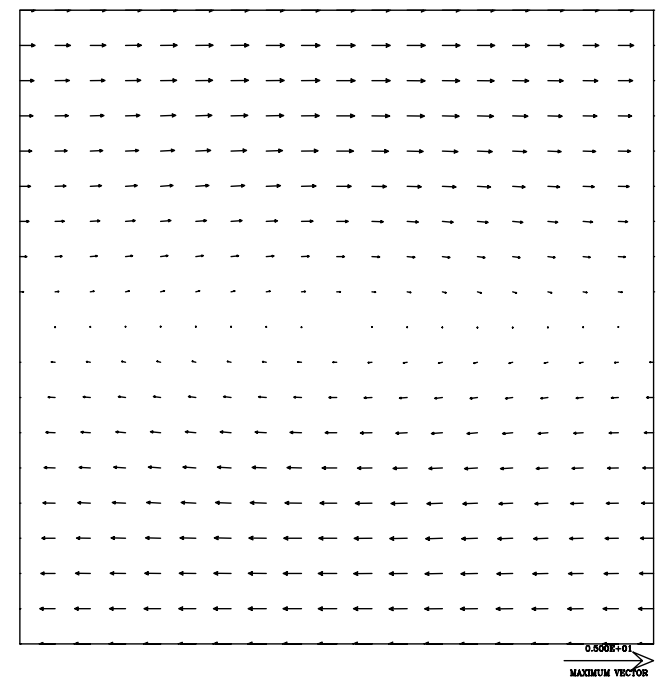

Figure 3. Perturbed magnetic field.

The equilibrium is defined by $B_{\mathrm{eq}}=\frac{\mathrm{d} \psi_{\mathrm{eq}}}{\mathrm{d} x} e_{y}=\operatorname{th}(\alpha x) e_{y}, V_{\mathrm{eq}}=0$ (then $S_{e}=u_{e}=g_{e}=0$ in Sect. 1). We also assume as in [8] symmetry conditions for all unknowns at $x=0$ and we consider a grid of 39 points $(\mathrm{N}=$ $39)$ on $] 0,1 / 2\left[\right.$, defined by $x_{i}=-1 / 4(\cos (i \pi /(N-1))-1)$.

In fusion experiments, the temperature $T$ of the plasma is slowly increased, at a slow diffusion time scale. The bifurcation parameter $S$ being considered as an increasing function of $T$, we are interested in the existence of bifurcations from a given equilibrium when $S$ is increased from low values. A bifurcation point corresponds necessarily to a value $S_{0}$ such that the kernel of $F_{U}\left(S_{0}, 0\right)$ is non trivial (otherwise, by the implicit function theorem, the trivial branch is the only one in a neighbourhood of $\left(S_{0}, 0\right)$ ). To apply Theorem 2, we want to find values $S_{0}$ such that the kernel is one-dimensional. First, we choose fixed values of the parameters $\alpha$ and $\wp_{R}\left(\alpha=4, \wp_{R}=0.2\right)$. Then, for each length $L$ in the periodic $y$ direction, the linear problem is decomposed (see Sect. 3) in a sequence of 1D problems, indexed by the integer $m$, with $k=2 \pi / L$. For each value of $k$ and $m$, the corresponding 1D problem only depends of the product $(m k)$. Then, for each value of $k \in \mathbb{R}$, we note by $S(k)$ the smallest value strictly positive of $S$ for which the 1D problem with $m=1$ has a non trivial solution. For a given length $L$, we obtain then a non trivial kernel of $F_{U}(S, 0)$ for each value of the parameter $S$ equal to $S(m k)$, with $k=2 \pi / L$ and for any integer $m 1$; the lowest value $S_{0}$ is then the infimum of this sequence $S(m k)$, for $m 1$. As $L$ is physically large (then $k$ small), the lowest value $S_{0}$ corresponding to that $L$ is not far from the minimum value $S\left(k_{0}\right)$ of the previously defined function $S(k)$, obtained for some value $k=k_{0}$.

Figure 1 is the curve of the quantity $S$ considered as depending of the wave number $k$.

Figure 2 represents the velocity field for a special value $k_{0}$ of $k$ which corresponds to the minimum of the curve $S(k)$ of Figure 1.

Figure 3 represents the magnetic field for a special value $k_{0}$ of $k$ which corresponds to the minimum of the curve $S(k)$ of Figure 1. 


\section{REFERENCES}

[1] A. Boussari, Etude des instabilités tearing avec résistivité variable. Ph.D. thesis, University of Clermont-Ferrand, France (1996).

[2] X.L. Chen and P.J. Morrison, Nonlinear interactions of tearing modes in the presence of shear flow. Institute for fusion studies, University of Texas (1991).

[3] M.G. Crandall and P.H. Rabinowitz, Bifurcation for simple eigenvalues. J. Funct. Anal. 8 (1971) 321-340.

[4] C. Foias and R. Temam, Remarque sur les équations de Navier-Stokes stationnaires et les phénomènes successifs de bifurcation. Ann. Scuola Norm. Sup., Pisa Cl. Sci. Ser. IV 5 (1978) 29-63.

[5] R. Grauer, Nonlinear interactions of tearing modes in the vicinity of a bifurcation point of codimension two. Phys. D 35 (1989) 107.

[6] J. Necas, Les méthodes directes en théorie des équations elliptiques. Masson, Paris (1967).

[7] R.D. Parker, Nonlinear behaviour of the resistive tearing instability in plasmas. Thesis, Australian National University (1987).

[8] B. Saramito, Analyse mathématique et numérique de la stabilité d'un plasma. Thèse d'État, Université Paris VI, France (1987).

[9] B. Saramito, Stabilité d'un plasma : modélisation mathématique et simulation numérique. Masson (1994).

[10] B. Saramito and E. Maschke, Bifurcation of steady tearing states. Int. Workshop on Magn. Reconn. and Turb., Cargèse (1985), Éditions de Physique, Les Ulis (1985).

[11] J. Simon, Régularité de la composée de deux fonctions et applications. Boll. Un. Mat. Ital. B (5) 16 (1979) 501-522. 